\title{
LOS MODELOS ORGANIZATIVOS DE TRAPEROS DE EMAÚS EN EL ESTADO Y SU RESPUESTA A LA CRISIS ${ }^{1}$
}

\author{
POR \\ Juan Carlos PÉREZ DE MENDIGUREN ${ }^{2}$ y \\ Unai VILLALBA ${ }^{3}$
}

\section{RESUMEN}

A pesar de su inspiración común en el movimiento impulsado por el Abbé Pierre en Francia en 1949, en la actualidad existen importantes diferencias entre los grupos de Traperos de Emaús. Este artículo pretende clasificar los diversos grupos presentes en el Estado español y analizar si sus características particulares les han llevado a plantear diferentes estrategias frente a la crisis. Para ello primero proponemos una tipología basada en el análisis de cinco variables (forma jurídica, existencia de vida en comunidad, liderazgo y participación, crecimiento, orientación de la acción) que ayuden a dibujar el modelo organizativo de los diversos grupos de Emaús en el Estado; lo que nos lleva a clasificarlos en cuatro categorías (Comunitaria, Fundación, Empresa Social, Mixta).

En general, las diferencias entre estos modelos son previas a la crisis económica, pero hemos observado que también hay diferencias en las respuestas a la misma. Aunque todavía es pronto para poder establecer una tipología de respuestas a la crisis, sí podemos subrayar tentativamente algunas claves que parecen contrastar las diferencias entre los modelos propuestos. Creemos que es importante comprender estos diferentes modelos y sus estrategias de respuesta, ya que todas ellas están presentes en otras entidades o empresas sociales

\footnotetext{
${ }^{1}$ Trabajo elaborado en el marco del Grupo de Investigación sobre Seguridad Humana, Desarrollo Humano Local y Cooperación Internacional, del sistema universitario vasco (GIU12/68)

${ }^{2}$ Departamento de Organización de Empresas. Universidad del País Vasco UPV-EHU. Dirección de correo electrónico: juancarlos.perezdemendiguren@ehu.es

${ }^{3}$ Departamento de Economía Aplicada II. Universidad del País Vasco UP-EHU. Dirección de correo electrónico: unai.villalba@ehu.es
}

REVESCO No 113 - MONOGRÁFICO: Crisis económica actual y sus posibles repercusiones en la economía social - ISSN: 1885-8031 - www.ucm.es/info/revesco

http://dx.doi.org/10.5209/rev_REVE.2014.v113.43381

Fecha de recepción: 12/07/2013

Fecha de aceptación: 28/11/2013 
vinculadas a la Economía Social y al Tercer Sector, y por tanto, ayudan a comprender cuáles pueden ser las claves de futuro de este tipo de Economía Alternativa.

Palabras clave: Economía Social, Economía Solidaria, Crisis, Empresas Sociales, Inserción sociolaboral, Empleo.

Claves Econlit: L39, M14, P49.

\title{
THE ORGANIZATIONAL MODELS OF EMAUS RAGPICKERS IN THE STATE AND ITS RESPONSE TO CRISIS
}

\begin{abstract}
Despite their common inspiration driven by the Abbé Pierre movement in France in 1949, today there are significant differences between the ragpickers groups. This article aims to classify the various groups in the Spanish state and analyze whether their particular characteristics have led them to propose different strategies to deal with the crisis. This first propose a typology based on the analysis of five variables (legal form of life on community leadership and participation, growth, action orientation) to help draw the organizational model of the various groups of Emaus in the state, which leads us to classify them into four categories (Community Foundation, Social Enterprise, Mixed).

In general, the differences between these models are previous to economic crisis, but we have observed that there are differences in the responses to it. Although it is still early to establish a typology of responses to the crisis, we can tentatively highlight some keys that seem to contrast the differences between the proposed models. We believe it is important to understand these different models and response strategies, as all of them are present in other social institutions or companies related to the Social Economy and the Third Sector, and therefore help to understand what might be the key to future this type of Alternative Economy.
\end{abstract}

Keywords: Social Economy, Solidarity Economy, Crisis, Social Enterprise, Social and labor insertion, Jobs.

REVESCO $\mathrm{N}^{\circ} 113$ - MONOGRÁFICO: Crisis económica actual y sus posibles repercusiones en la economía social - ISSN: 1885-8031 - www.ucm.es/info/revesco 


\section{INTRODUCCIÓN}

La primera comunidad de Emaús se puso en marcha en 1949 impulsada por el Abbé Pierre en Francia, pero en la actualidad se trata de un movimiento internacional presente en varios países de diferentes continentes. En el Estado español las primeras comunidades y grupos de Emaús se formaron a inicios de los años 70 en Bizkaia y Navarra, y hoy en día se puede encontrar presencia de Emaús en unas 35 ciudades y pueblos, agrupados en torno a siete grupos principales.

Estos siete grupos matrices forman el núcleo central de colectivos de Traperos de Emaús en el Estado español y cada uno, con la sola excepción de Emáus Fundación Social, tiene un ámbito de intervención que se circunscribe al espacio local o provincial (ver cuadro 1). Aunque cada grupo ha mantenido una forma de funcionamiento autónoma, gran parte de la gestión legal recaía sobre una estructura común hasta el año 1997. A partir de ese año, y aun manteniendo en común los valores y principios fundamentales del movimiento, cada colectivo marca un camino independiente, con un nivel de coordinación inter-grupos bajo, que acaba dando lugar a diferentes resultados organizativos que son objeto de estudio en este trabajo. Posteriormente, en el 2006, estos siete grupos retomaron cierto grado de coordinación estatal, firmaron una declaración de identidad común que incide en los aspectos centrales de la filosofía de Emaús ${ }^{4}$, y que proporciona a los grupos firmantes el reconocimiento mutuo de pertenencia al colectivo Emaús en el Estado.

Cuadro 1: Grupos Emaús en el Estado español

\begin{tabular}{|l|c|l|c|c|}
\hline Denominación & Provincia & $\begin{array}{l}\text { Forma } \\
\text { societaria }\end{array}$ & $\begin{array}{l}\text { Año } \\
\text { fundación }\end{array}$ & $\begin{array}{l}{ }^{(1)} \mathbf{N}^{\mathbf{0}} \\
\text { personas }\end{array}$ \\
\hline Emaús Bilbao Sociedad Cooperativa & Bizkaia & $\begin{array}{l}\text { Sociedad } \\
\text { Cooperativa }\end{array}$ & 1971 & 95 \\
\hline Traperos de Emaús Navarra & Navarra & Fundación & 1972 & 206 \\
\hline Emaús Fundación Social Grupo & Gipuzkoa & $\begin{array}{l}\text { Fundación } \\
\text { Social }\end{array}$ & 1980 & 115 \\
\hline Drapaíres de Emaús & Barcelona & $\begin{array}{l}\text { Asociación/ } \\
\text { Cooperativa }\end{array}$ & 1980 & 14 \\
\hline Traperos de Emaús Murcia & Murcia & Asociación & 1995 & 73 \\
\hline Traperos de Emaús Granada & Granada & Asociación & 1998 & 5 \\
\hline Traperos de Emaús Asociación Comunitaria & Araba & Asociación & 2002 & 26 \\
\hline
\end{tabular}

1. Estas cifras se refieren al número de personas empleadas en el 2011, salvo Granada referida a 2012. Todos los grupos movilizan además a un importante grupo de personas voluntarias con grados de implicación y asunción de roles muy variable entre ellas.

\footnotetext{
${ }^{4}$ http://www.emaus.org/declaracion.html REVESCO $\mathrm{N}^{\circ} 113$ - MONOGRÁFICO: Crisis económica actual y sus posibles repercusiones en la economía social - ISSN: 1885-8031 - www.ucm.es/info/revesco
} 
Adicionalmente, y debido a la lógica de creación y expansión descentralizada y autónoma de las experiencias, existen en el Estado al menos otros dos grupos que se autodenominan Traperos de Emaús o tienen un origen histórico ligado al movimiento pero que no están en relación con el colectivo estatal, y tampoco participan en Emaús Internacional. $^{5}$

El objetivo de este artículo es doble: primero estudiar los modelos organizativos de los grupos de Traperos de Emaús y establecer una tipología, y segundo observar si existen impactos y/o respuestas diferenciadas a la crisis económica en base a esa tipología. Ahora bien, como indicaremos en el siguiente apartado, la perspectiva de análisis más concreta ha sido desde la ubicación de los grupos como entidades o empresas de la Economía Solidaria. Si bien algunos grupos se observan a sí mismos más como asociaciones y/o comunidades que como empresas, otros sí se reconocen en este ámbito y por ejemplo participan en espacios como REAS (Red de Economía Alternativa y Solidaria). En cualquier caso, creemos que es pertinente esta perspectiva, ya que la totalidad de los grupos tiene actividades productivas "que buscan la independencia económica a través del trabajo (especialmente de recuperación, reutilización, y reciclaje de residuos)", principalmente a través de cooperativas o empresas de inserción, que tanto en el ámbito español como en el europeo (Chaves y Monzón, 2012; Defourny y Nyssens 2006), son normalmente ubicadas como empresas sociales en el marco de la Economía Social y Solidaria.

Las herramientas metodológicas utilizadas en el estudio han sido la revisión documental (interna y pública), entrevistas (semi-estructuradas, en profundidad, individuales y grupales, y a informantes clave), observación y visita a centros, así como recogida de datos e indicadores en particular para el caso de Araba, Gipuzkoa, Navarra y Bizkaia a través del Sistema de Indicadores de Auditoría Social propuesto por REAS. En un primer momento se elaboró un borrador que fue discutido en seminarios académicos y enviado a los grupos Emaús, en un segundo momento ese borrador fue ajustado y precisado después de las visitas y entrevistas, y posteriormente se les ha devuelto el documento final.

\footnotetext{
${ }^{5}$ Estos grupos son la Asociación Emaús en Altea y los Traperos de Emaús Madrid. A efectos del trabajo realizado para este artículo únicamente han sido considerados los grupos Emaús que componen el colectivo estatal que se refleja en el cuadro 1. No entramos a valorar si el resto deberían o no ser considerados como grupos Emaús. Nuestro criterio para no incluirlos es puramente analítico ya que consideramos que su inclusión no aportaría elementos (historia, dinámica o estructura organizativa) novedosos que no hayan quedado suficientemente recogidas en el conjunto de grupos que ya incluimos en el análisis.
}

REVESCO No 113 - MONOGRÁFICO: Crisis económica actual y sus posibles repercusiones en la economía social - ISSN: 1885-8031 - www.ucm.es/info/revesco 


\section{TRAPEROS DE EMAÚS EN LOS DEBATES SOBRE LA ECONOMIA SOLIDARIA}

A pesar de que la actividad productiva es sólo una parte de la actividad de los grupos de Emaús, desde un punto de vista conceptual el análisis de su devenir organizativo está directamente vinculado a los debates teóricos sobre la Economía Solidaria y la Empresa Social (o el Emprendimiento Social), términos que pretender dar cuenta de un crisol multiforme de organizaciones que resisten y cuestionan las narrativas tradicionales sobre los procesos económicos y la racionalidad de las empresas.

A pesar de la todavía escasa evidencia empírica sobre el tema, el interés por estas empresas se acentúa a la vista de la naturaleza de los objetivos sociales que se proponen (normalmente relacionados con la lucha contra la exclusión social y la pobreza), y de su aparente capacidad para conciliarlos con cierto grado de rentabilidad financiera, o al menos, con la capacidad de sostener financieramente la actividad productiva realizada. Pero también por el carácter innovador de las estrategias e instrumentos de gestión utilizados, por la combinación de recursos que son capaces de movilizar, y por la capacidad potencial de replicar sus modelos de negocio tanto a nivel de escala como en diferentes ámbitos geográficos.

Sin embargo, y como cabría esperar, Empresa Social y Emprendimiento Social son conceptos controvertidos sobre los que existen múltiples definiciones construidas desde perspectivas teóricas dispares. Aunque su discusión detallada desborda las posibilidades de este artículo, Díaz y Marcuello (2010) analizan su significado en el caso español, mientras que un buen análisis de las diferencias entre los enfoques europeo y norteamericano puede encontrarse en Kerlin (2006, 2010) o Defourny y Nyssens (2010). Por su parte Peattie y Morley (2008) presentan un detallado estado de la cuestión sobre los debates teóricos y prácticos asociados al tema, y proponen una agenda de investigación sobre los mismos. La complejidad conceptual aumenta por el hecho de que aunque habitualmente Empresa Social y Emprendizaje Social (Social Entrepreneurship) se toman como sinónimos, en el debate conceptual no pueden considerarse como tales ${ }^{6}$.

\footnotetext{
${ }^{6}$ Emprendizaje social es un término más general que englobaría al anterior, pero es además pone su acento en: (1) personas individuales, visionarias, y no tanto en las organizaciones; (2) el empresario social como alguien lidera procesos de innovación social; (3) énfasis en la replicabilidad, en el impacto a gran escala y finalmente (4) REVESCO $\mathrm{N}^{\circ} 113$ - MONOGRÁFICO: Crisis económica actual y sus posibles repercusiones en la economía social - ISSN: 1885-8031 - www.ucm.es/info/revesco
} 
A pesar de la diversidad de enfoques, existe cierto consenso sobre el surgimiento de una narrativa dominante sobre el tema que algunos autores denominan como del EmpresarioHéroe (Nicholls, 2010) o narrativa del cambio social armónico (Dey y Steyaert, 2010). Según esta visión, de la mano de estas organizaciones (y del carácter emprendedor e innovador de sus promotores) se abriría la posibilidad de impulsar una suerte de cambio social de carácter armónico hacia una forma de capitalismo inclusivo o filantrocapitalismo (Bishop y Green, 2008), basado en la denominada creación de valor compartido (Porter y Kramer, 2011) ${ }^{7}$. Esta narrativa además se estaría imponiendo a las visiones de la Empresa Social basadas en modelos comunitarios y en una lógica de acción colectiva ligada a la justicia social y el empoderamiento de las personas (Nicholls, 2010).

Por otra parte es necesario indicar que estos conceptos no tienen el mismo significado en todos los países o ámbitos geográficos. Mientras que en algunos lugares (caso del Estado Español) es poco habitual encontrar organizaciones que se autodenominen Empresas Sociales (Díaz y Marcuello 2012), en otros (como el Reino Unido) éstas se han constituido en elementos centrales de los procesos de reforma del Estado del Bienestar (Ridley-Duff y Bull, 2011; Teasdale 2012). Además, y a diferencia de los países de habla inglesa, en gran parte de la Europa continental las Empresas Sociales engarzan con la tradición de la Economía Social (Defourny y Nyssens 2010 y 2006; Chaves y Monzón 2012; Spear 2006), o con lo que se viene denominando en los últimos años Economía Solidaria (Coraggio 2011, Laville 2004).

En el caso de ésta última, aunque habitualmente caracterizada como integrada en el tronco común de la Economía Social (Enjolras 2010; Chaves y Monzón 2012), su cuerpo teórico reúne conceptos e ideas con origen en Latinoamérica y Europa (Da Ros, 2007; Pérez de Mendiguren et. al, 2009). Como indican Martínez y Álvarez (2008), la Economía Solidaria designa por un lado un enfoque teórico sobre la economía que parte de una crítica al modo de producción capitalista y su concepción de "lo económico" (Coraggio 2011). Propone una reconceptualización de la lógica económica desde una perspectiva humanista situando a las personas y a la reproducción ampliada de la vida en el centro de los procesos de económicos.

\footnotetext{
la idea de que el emprendizaje social pueden ocurrir en cualquier sector, incluidos el sector privado (empresas convencionales) y el sector público.

7 Esta propuesta ha generado un encendido debate entre algunos teóricos defensores del enfoque de la Responsabilidad Social Corporativa (RSC), que la consideran un invento innecesario por entender que es la misma idea original de la RSC con otro nombre. Ver por ejemplo la critica de Henk Campher en http://corporatesocialreality.net/2011/02/25/, o, desde un punto de vista un diferente, la de Michael Sadowski en: http://www.sustainability.com/blog/what-s-new-about-creating-shared-value
}

REVESCO No 113 - MONOGRÁFICO: Crisis económica actual y sus posibles repercusiones en la economía social - ISSN: 1885-8031 - www.ucm.es/info/revesco 
Pero a su vez la Economía Solidaria nombra a un tipo específico de organización basada en la democracia y el empresariado colectivo. Así, en el caso del Estado Español, muchas de los emprendimientos que en el resto de Europa serían catalogados como Empresas Sociales (por ejemplo las empresas de inserción o WISE- Work Integration Social Enterprises), han construido su identidad colectiva como entidades de Economía Solidaria agrupadas entorno a REAS.

Además, y en contraposición a la narrativa del cambio social armónico, las organizaciones de Economía Solidaria hacen su camino en medio de una constante necesidad de (re)construcción de su legitimidad, e identidad. Su carácter de entidades frontera las coloca en una posición de tensión creativa y conflictiva entre: el mercado, los movimientos sociales y el estado; entre las lógica de las solidaridad, la donación y la recuperación de costes; entre la participación y la eficiencia en la prestación de servicios; entre el voluntariado y la profesionalidad; entre el compromiso social y el empleo remunerado; entre las organizaciones sociales y las empresas.

En un estudio sobre la caracterización de los procesos de crisis enfrentados por las Empresas de Economía solidaria en el Estado Español, Argentina y Brasil, Guridi et al. (2011) concluyen que éstas tienen que ver con la dificultad para mantener la identidad, la legitimidad y la coherencia organizativa en situaciones inducidas por factores de orden externo e interno. Entre los primeros destacan los que dificultan la adecuación del modelo organizativo a los cambios en el entorno social e institucional, o a las exigencias de la competencia en el mercado. Entre los segundos aparecen aquellos asociados a las dinámicas de crecimiento y control de la organización, la gestión de los liderazgos, o las crisis en los modelos de participación. El caso de las organizaciones Traperos de Emaús en el Estado Español, presentado a continuación, proporciona un estudio de caso particular de las dinámicas aquí descritas.

\section{MODELOS ORGANIZATIVOS DE TRAPEROS DE EMAÚS: UNA TIPOLOGÍA}

\subsection{Condicionantes del modelo organizativo}

Todavía en clave histórica, y antes de entrar en el análisis de las tipologías, consideramos conveniente plantear dos condicionantes del entorno que influyen en la evolución de los modelos organizativos y otros dos internos. El primer condicionante del

REVESCO No 113 - MONOGRÁFICO: Crisis económica actual y sus posibles repercusiones en la economía social - ISSN: 1885-8031 - www.ucm.es/info/revesco 
entorno tiene que ver con el advenimiento de la democracia a finales de los 70, la proclamación del estado laico y el boom del asociacionismo ciudadano y político. En ese momento aparecen (o toman carta de legalidad) una plétora de organizaciones sociales y asociaciones que tienen como objetivo la lucha por la justicia social y/o contra la pobreza y sus manifestaciones. En ese contexto, Traperos de Emaús inicia un recorrido propio, después de haber nacido en relación con la Iglesia, quien concentraba hasta el momento la mayoría del trabajo de asistencia que no proporcionaba el Estado.

El segundo condicionante es el progresivo desarrollo del Estado del Bienestar y su evolución, dispar entre comunidades según avanzaba la descentralización del Estado, que ha ido modificando el papel de los Traperos de Emaús en cuanto a su relación con las políticas sociales. Mientras que su objetivo de "trabajo con los que más sufren y lucha contra las causas que generan el sufrimiento" ha permanecido inalterado, la progresiva asunción por parte de las comunidades autónomas de las políticas de asistencia social, servicios sociales y promoción del bienestar ha ido modificando el rol que juegan Traperos de Emaús y su relación con los organismos públicos. Por una parte, muchos de los recursos sociales de asistencia empezaron a proveerse desde el sector público, y por otra, los propios grupos de Emaús empezaron a jugar un rol importante colaborando e incidiendo en el diseño y gestión de algunas de las nuevas políticas contra la exclusión social. Varios de los grupos ofrecen servicios en el ámbito de la acogida, la orientación y formación en habilidades sociales y oficios, y en la integración laboral directa en sus "empresas de inserción laboral" y "centros especiales de empleo". Estas actividades se financian en base a una combinación de ingresos mercantiles, contratos con administraciones y subvenciones estatales.

Por su parte, los dos condicionantes de orden interno están estrechamente ligados entre sí, se trata del importante crecimiento de los grupos y la posterior ruptura del modelo organizativo comunitario originario. En diferentes momentos según la provincia, se produjo un importante crecimiento orgánico en los grupos Emaús de Bilbao, San Sebastián y Pamplona, de la mano de los sucesivos contratos con instituciones públicas para la recogida selectiva de residuos y actividades de recicle, o de la puesta en marcha de iniciativas de trabajo propias que aumentaban la diversidad de situaciones de las personas en relación a Emaús. Este crecimiento y la continua incorporación de miembros van a tener importantes consecuencias en el modelo de organización interna y una crisis que terminará con el modelo comunitario originario. Al tiempo que los grupos se desarrollaron a nivel productivo, la 
comunidad a nivel físico no podía crecer en la misma medida. En el caso de Pamplona la vida en comunidad pasa a ser una opción voluntaria (que se mantiene hasta el día de hoy), y aunque permanece en el centro del imaginario colectivo del grupo y su cultura organizativa, los asuntos generales pasan a gestionarse en el marco de una fundación con estructura separada de la comunidad. Si bien en la práctica, tal y como se explica más adelante, el grupo insiste en la no disociación de sus labores productivas y sociales, y por ende, tampoco entre las estructuras de la fundación y la comunidad. En Bilbao y San Sebastián, tanto por la marcha de algunas personas referenciales como por la búsqueda de una nueva orientación de las actividades, las comunidades se disuelven en diferentes momentos, tomando la determinación de separar el ámbito social del ámbito productivo-empresarial, y creando una fundación en San Sebastián y una Cooperativa en Bilbao.

\subsection{Tipología de grupos Emaús en el Estado}

Los cuatro modelos organizativos que presentamos a continuación (Modelo Comunitario, Modelo Fundación, Modelo Empresa Social, Modelo Mixto) comparten los principios del movimiento de Traperos de Emaús y su ética. Sin embargo, la diferente forma en que se han gestionado las presiones internas y externas y su evolución, han dado lugar a diferentes resultados organizativos. Es importante hacer notar que los modelos que presentamos a continuación deben ser considerados al estilo de los "tipos ideales" weberianos, es decir, que son un constructo teórico que permite comparar y caracterizar los comportamientos, y no una descripción exacta de la realidad de ningún grupo en concreto. De hecho, la realidad es más compleja que la imagen dibujada por los modelos, y algunos grupos presentan características que podrían ser encuadradas en más de un modelo.

La generación de estos modelos está basada en el análisis de documentos propios de las organizaciones, y contrastada después en entrevistas en profundidad con miembros fundadores de cada uno de los colectivos, conocedores de su historia, evolución y perspectivas actuales. Los modelos se han generado agrupando características en torno a las siguientes variables: 1) forma jurídica; 2) existencia de comunidad de convivencia; 3) formas de liderazgo y participación, y modelo de gobernanza; 4) razones para el crecimiento de la organización; 5) orientación global de la acción. Las características de cada una de estas variables que se muestran en el Cuadro 2 se explican para cada uno de los modelos a continuación.

REVESCO No 113 - MONOGRÁFICO: Crisis económica actual y sus posibles repercusiones en la economía social - ISSN: 1885-8031 - www.ucm.es/info/revesco 
Cuadro 2. Modelos de Traperos de Emaús en el Estado.

\begin{tabular}{|l|l|l|l|l|}
\hline $\begin{array}{l}\text { MODELOS / } \\
\text { Variables }\end{array}$ & COMUNITARIO & FUNDACIÓN & $\begin{array}{l}\text { EMPRESA } \\
\text { SOCIAL }\end{array}$ & MIXTO \\
\hline Forma Jurídica & Varias & Fundación & Cooperativa & $\begin{array}{l}\text { Fundación/ } \\
\text { Asociación }\end{array}$ \\
\hline $\begin{array}{l}\text { Existencia } \\
\text { Comunidad }\end{array}$ & Sí, nuclear & No, pero la hubo & No, pero la hubo & Sí, opcional \\
\hline $\begin{array}{l}\text { Liderazgo, } \\
\text { Participación }\end{array}$ & Carismático & $\begin{array}{l}\text { Personalista/ } \\
\text { Profesionalizado }\end{array}$ & $\begin{array}{l}\text { Colectivo/ } \\
\text { Profesionalizado }\end{array}$ & $\begin{array}{l}\text { Carismático/ } \\
\text { Colectivo }\end{array}$ \\
\hline $\begin{array}{l}\text { Crecimiento } \\
\text { Orientación } \\
\text { Acción }\end{array}$ & Orgánico & Inducido/ Impacto & $\begin{array}{l}\text { Estratégico/ } \\
\text { Competitivo }\end{array}$ & $\begin{array}{l}\text { Orgánico/ } \\
\text { Estratégico }\end{array}$ \\
\hline $\begin{array}{l}\text { Grupos } \\
\text { Correspondientes }\end{array}$ & $\begin{array}{l}\text { Drapaíres de } \\
\text { Emaús (Sabadell) }\end{array}$ & $\begin{array}{l}\text { Emaús Fundación } \\
\text { Social (Gipuzkoa) }\end{array}$ & $\begin{array}{l}\text { Emáus Bilbao Soc. } \\
\text { Cooperativa } \\
\text { Traperos Emaús } \\
\text { Vitoria }\end{array}$ & $\begin{array}{l}\text { Traperos Emaús } \\
\text { Navarra y Murcia }\end{array}$ \\
\hline
\end{tabular}

1. No hemos querido clasificar al grupo de Granada porque se trata de un grupo pequeño, de unas cinco personas dedicadas actualmente al autoempleo, y porque se encuentran en proceso de reestructuración.

\subsubsection{Modelo Comunitario}

Aunque históricamente pudiera haber habido otros, en la actualidad, el grupo correspondiente a este modelo es Drapaíres de Emaús de Sabadell. La forma jurídica que adopta este colectivo es la de asociación apartidista, aconfesional y no lucrativa. Es en esta asociación en la que se desarrollan la comunidad y los grupos de amigos y voluntarios. Complementariamente, el grupo mantiene en su seno una cooperativa de reinserciónsociolaboral que da cobertura legal a sus actividades productivas de recogida, reciclado, reparación y venta de residuos y productos de comercio justo ${ }^{8}$; y una fundación que canaliza los esfuerzos de solidaridad y cooperación con otros colectivos en el ámbito estatal e internacional. Así que cada uno de los tres pilares de su acción (solidaridad, trabajo, comunidad) se ha convertido en una forma jurídica (fundación, cooperativa, asociación). Uno de los aspectos reseñables de la estructura legal del grupo es el carácter subsidiario de las formas legales adoptadas. El mantenimiento de la coherencia colectiva con los objetivos y la conservación de la comunidad son el centro alrededor del que pivotan el resto de elementos y decisiones que tienen cierto carácter subordinado a lo anterior. En ese sentido, la estructura legal bajo la que se ampara el colectivo no determina la lógica de funcionamiento ni los objetivos del mismo.

\footnotetext{
${ }^{8}$ La ley de Empresas de Inserción (Ley 44/2007) obliga a que la entidad promotora de la inserción y la que realiza la actividad económica que sirve de base para la inserción por el empleo sean dos entes jurídicos diferenciados. De esta forma todos los grupos de Emaús han optado por la creación de empresas de inserción instrumentales a sus objetivos.
}

REVESCO No 113 - MONOGRÁFICO: Crisis económica actual y sus posibles repercusiones en la economía social - ISSN: 1885-8031 - www.ucm.es/info/revesco 
En este modelo la vida en comunidad es evidentemente fundamental, y constituye el núcleo de toda su visión y organización. Este modelo responde a la idea originaria de comunidad de vida y trabajo, autosuficiente y bajo la responsabilidad colectiva y mancomunada sobre el bienestar de los miembros que la componen, así que todos los recursos y dinero se gestionan comunitariamente. En ese sentido enfatizan la capacidad de generar recursos propios a través del trabajo, manteniendo un alto nivel de independencia frente a un sistema que, según señalan, genera situaciones de injusticia y pobreza para la mayoría de la población mundial. Se presentan como una opción de vida sencilla en comunidad, viviendo del trabajo de traperos de forma coherente con las ideas, principios y objetivos que persiguen.

Con respecto al tipo de liderazgo y formas de participación que vertebran el grupo, se puede decir que son fundamentalmente de tipo carismático. El mantenimiento de la coherencia y la opción por un modelo de vida comunitario son las fuentes del liderazgo, que se refuerza por la permanencia de varias de las personas que iniciaron el proyecto.

Este aspecto del liderazgo carismático y comprometido está ligado al carácter utópico del modelo organizativo y la orientación de sus acciones, ya que la capacidad de generar el impacto de transformación social que se busca pasa por el mantenimiento de la coherencia del grupo. Ahora bien, el grupo está constantemente buscando la interacción con otras organizaciones y redes sociales para maximizar ese potencial transformador y de compromiso con la justicia social.

Bajo estos parámetros, el modelo de crecimiento de la organización y/o de la multiplicación de su impacto (scaling-up) puede calificarse como orgánico. Lo relevante no es hasta dónde se es capaz de llegar, sino que el interés por crecer y generar mayor impacto no acabe poniendo en peligro la coherencia y supervivencia del propio grupo. Esto explica el hecho de que la comunidad pueda tener en algún momento capacidad de acogida excedentaria que no utiliza.

\subsubsection{Modelo Fundación}

El surgimiento del modelo fundación responde a una lógica de respuesta a la evolución de las políticas públicas de intervención socio-laboral, caso que podemos asociar principalmente al grupo de San Sebastián, Emaús Fundación Social. En los últimos 15 años esta fundación se ha convertido en una organización importante que ha crecido tanto en

REVESCO No 113 - MONOGRÁFICO: Crisis económica actual y sus posibles repercusiones en la economía social - ISSN: 1885-8031 - www.ucm.es/info/revesco 
número de trabajadores como en número y tipo de proyectos gestionados, así como incluso en implantación territorial. De hecho, es el único grupo Emaús que ha trascendido las fronteras provinciales para implantarse en otras localidades de provincias no limítrofes. La fundación gestiona centros de acogida temporal para personas sin recursos, alojamientos de inserción de larga estancia, centros de formación y orientación al empleo, acompañamiento en el puesto de trabajo y prospección e intermediación laboral. Además, la fundación es promotora y socia principal de: dos empresas de inserción y una cooperativa de iniciativa social dedicadas a la gestión de residuos y textiles; una empresa de inserción dedicada a la comercialización de productos de comercio justo; y una empresa dedicada a la promoción de empresas e iniciativas de autoempleo.

Desde el punto de vista de la forma jurídica, la figura determinante es la de fundación. Cabe destacar que las fundaciones son figuras pensadas para gestionar patrimonio en persecución de un fin social, y no tanto para articular dinámicas de trabajo o participación entre personas. Este tipo de figura, en el que el elemento central es la optimización de la gestión patrimonial, impulsa la adopción de formas de trabajo de carácter más profesionalizado, pero no es tan favorecedor a la hora de generar dinámicas de identificación entre "profesionales" y "beneficiarios" y a la hora de promover la participación.

Es importante indicar que recientemente se ha separado la estructura del grupo Emaús de San Sebastián. Por un lado estaría la cooperativa, relativamente pequeña y dedicada por ahora al autoempleo, por lo que no podemos asemejarla a la cooperativa de Bilbao ya que no trabaja con empresas de inserción. Por otro lado, la parte social, de casas de acogida y empresas de inserción, estaría a cargo de la Fundación.

En cuanto al liderazgo, en este modelo también se observa que uno de los elementos de cohesión es la permanencia en la organización de algunas personas históricas y carismáticas que pusieron en marcha la experiencia. Pero el tipo de liderazgo tiene un matiz más personalista (influencia de personas concretas y su experiencia en la organización) y profesionalizado, asociado a la eficacia en la producción de resultados y gestión de equipos. En este sentido, la autoridad funcional (derivada de la posición en la estructura organizativa) tiende a desempeñar un mayor papel que la autoridad moral.

Respecto a la orientación de su acción, este modelo tiene un carácter más asistencial, derivado del papel que juega en la orquestación y gestión de las políticas públicas y los REVESCO No 113 - MONOGRÁFICO: Crisis económica actual y sus posibles repercusiones en la economía social - ISSN: 1885-8031 - www.ucm.es/info/revesco 
servicios sociales, siendo limitada la capacidad del grupo para influenciar el diseño y la orientación de estas políticas. Esto es evidente por ejemplo en centros de acogida, ya que no se gestiona de manera autónoma desde la Fundación, sino que son fruto de una política concertada con los gobiernos provinciales: "son los servicios sociales de base de la Diputación los que valoran cada caso y determinan el programa adecuado para cada solicitante".

Como se dijo más arriba, se trata de un grupo que ha crecido considerablemente, siguiendo una lógica de crecimiento inducido, explicado por la necesidad de maximizar el impacto social de las políticas sociales orquestadas, y por el interés por aumentar la cobertura de manera que se llegue al mayor número posible de personas en situación de exclusión, o al mayor número de personas que conforman los grupos objetivo del resto de intervenciones o servicios: "queremos atender todos los casos posibles que se nos presenten, llegar a tantas personas en situación de exclusión como podamos".

Por último cabe mencionar brevemente que en la actualidad la idea de comunidad de convivencia está prácticamente ausente en el imaginario y filosofía de la fundación, a pesar de que en los orígenes del grupo sí existiera.

\subsubsection{Modelo Empresa Social}

Este modelo refleja la experiencia de Emaús Bilbao Sociedad Cooperativa a partir de 1997 cuando se incorporan a la organización un grupo nuevo de personas, que no habían participado en Emaús hasta entonces, y decidieron dar a la cooperativa un modelo autónomo que cambió su lógica inicial. Emaús Bilbao es una Sociedad Cooperativa de Iniciativa Social ${ }^{9}$, que nace agrupando como socios/as a trabajadores/as en procesos de inserción y trabajadores/as normalizados/as. La idea consistió en: "optar por un modelo de cooperativa para que los trabajadores y trabajadoras tuvieran responsabilidad patrimonial sobre la organización, y fueran a su vez corresponsables de las decisiones de la misma".

\footnotetext{
${ }^{9}$ El Decreto 61/2000 del 4 de Abril determina que el objeto de las Sociedades Cooperativas de Iniciativa Social será: a) la prestación de servicios asistenciales mediante la realización de actividades sanitarias, educativas, culturales u otras de naturaleza social; b) desarrollo de cualquier actividad económica que tenga por fin la integración laboral de las personas excluidas socialmente. Estas cooperativas deben de ser sin ánimo de lucro y por lo tanto cumplir los siguientes requisitos: a) No distribución de resultados positivos b) Interés al capital social no superior al interés legal del dinero; c) Desempeño gratuito de los cargos del Consejo Rector; d) Retribuciones a los socios trabajadores no podrán superar en más del $50 \%$ el convenio aplicable.
}

REVESCO No 113 - MONOGRÁFICO: Crisis económica actual y sus posibles repercusiones en la economía social - ISSN: 1885-8031 - $\underline{\text { www.ucm.es/info/revesco }}$ 
Además de las áreas de trabajo típicas de Emaús (empresas de inserción dedicadas a actividades tradicionales de traperos), la Cooperativa presta servicios de limpieza a comunidades de propietarios, oficinas y servicios públicos a través de dos nuevas empresas de inserción. Por otro lado, desde hace cuatro años se ha comenzado a trabajar en el sector de la movilidad sostenible, con la gestión de un servicio de préstamos de bicicletas que funciona ya en tres ayuntamientos vascos.

Con respecto a las variables que caracterizan este modelo organizativo, es interesante notar que la forma jurídica primordial es la cooperativa. Esta figura proporciona un modelo de empresa centrado en las personas en cuya base filosófica se encuentra la democracia económica y la corresponsabilidad. La cooperativa Emaús Bilbao es, a su vez, propietaria mayoritaria de cuatro empresa de inserción con personalidad jurídica propia (normalmente SL y SLU). Sin embargo su existencia es puramente instrumental, manteniéndose la cooperativa como centro neurálgico de la vida del colectivo. De hecho, tal y como subraya uno de los miembros del equipo de dirección: "la necesidad de gestionar múltiples figuras jurídicas, si acaso, complica la gestión, que sería mucho más sencilla si pudiera llevarse directamente desde la cooperativa".

Esta preponderancia del modelo cooperativo se entronca con el modelo de liderazgo que se persigue. Con este modelo se trata de pasar de un estilo de liderazgo de carácter personalista a otro de carácter colectivo que tenga como base la participación real de las personas que conforman el grupo. Esta idea supone uno de los caballos de batalla más importante para el grupo, y un elemento de permanente conflicto, ajuste y aprendizaje, ya que: "supone la articulación de espacios de decisión adecuados a las posibilidades y el interés de los diferentes grupos de personas que forman la organización, y que no parte de las mismas condiciones". Esta cuestión es particularmente relevante a la hora de implicar en el modelo de decisión a personas que se encuentran en diferentes momentos de su itinerario de inserción. Se manifiesta así un intento por generar una cultura organizativa que transmita valores cooperativos y solidarios de la organización a todas las personas que la forman, ya sean personal "técnico" o personas en "proceso de inserción". El desarrollo de un modelo de liderazgo colectivo se entiende como una fortaleza que cohesiona, y supone además una ruptura con la tradición de otros grupos Emaús que tienden a articularse alrededor de un líder sólido que convierte el grupo en su proyecto vital, y cuya permanencia supone un importante factor aglutinante, pero también puede generar excesiva dependencia.

REVESCO No 113 - MONOGRÁFICO: Crisis económica actual y sus posibles repercusiones en la economía social - ISSN: 1885-8031 - www.ucm.es/info/revesco 
En cuanto a la lógica de crecimiento de la organización, destacaríamos su visión estratégico/competitiva. La organización ha tenido un crecimiento muy importante, pasando de 5 trabajadores en 1997 a 95 en 2011, determinado por la promoción de las anteriormente citadas empresas de inserción ${ }^{10}$. Este crecimiento deriva en gran medida del marcado carácter productivo/mercantil de este modelo y de la estructura jurídica empresarial que marca su orientación. La cooperativa se rige bajo parámetros económicos para poder adecuarse a las exigencias mercantiles que se les plantean, y el espíritu empresarial, aun creando a veces problemas de identidad, está presente en muchas de sus decisiones. Hay que tener en cuenta que en el caso de Bizkaia, el nivel de competencia en el sector de actividad tradicional de Emaús es mayor que en otros lugares, lo que ha obligado a una mayor atención estratégica frente a las opciones de mercado, y a buscar en otros sectores de actividad, innovando desde el punto de vista productivo pero manteniéndose fieles a los principios de Emaús. Ahora bien, esta predominancia de la orientación productiva, no supone negar la co-existencia de lógicas asistenciales o utópicas en su seno.

Por último, cabe mencionar la inexistencia de una comunidad de base referencial, y aunque para algunas personas está en el imaginario colectivo, para otras sería tan sólo una opción práctica y transitoria de casa de acogida y empadronamiento para la obtención de ciertos derechos de ciudadanía para personas en riesgo de exclusión, y de modo funcional a la actividad laboral.

\subsubsection{Modelo Mixto}

Dentro de este modelo podemos ubicar dos grupos de referencia, Traperos de Emaús de Navarra y de Murcia. A pesar de que hay ciertas diferencias entre ambos grupos, también en la evolución histórica puesto que la experiencia en Navarra es bastante más dilatada, queremos comenzar señalando una característica común propia de este modelo: la existencia de una comunidad de vida que es de carácter opcional. En la actualidad, tan solo una pequeña parte de las personas de cada grupo viven en comunidad, generalmente personas "en procesos de inserción", y no tanto trabajadores/as "normalizados". Pero tal y como apuntan desde Navarra, habría que matizar esta realidad al menos por dos cuestiones: (i) la idea y el simbolismo de la comunidad es un elemento central del imaginario colectivo válido para

\footnotetext{
${ }^{10}$ Incluso en el 2012, temporalmente se llegó a los 400 trabajadores debido al Programa Sustapen, que sin embargo no se ha renovado en la actualidad.
}

REVESCO No 113 - MONOGRÁFICO: Crisis económica actual y sus posibles repercusiones en la economía social - ISSN: 1885-8031 - www.ucm.es/info/revesco 
todas las personas de Emaús, además de un espacio desde donde el que recrear formas alternativas de vida; (ii) la distinción entre "personas en inserción" y "normalizados", no es válida en muchos sentidos, y además, hay muchas personas que aun no durmiendo en la casa de acogida comunal, prácticamente "viven" en la comunidad. Aunque, por el contrario, también debemos reseñar que hay una corriente interna de personas que considerarían la comunidad como un cierto lastre para la actividad "empresarial" y laboral.

Respecto a la forma jurídica, en el caso navarro la organización central es una fundación, mientras que en el murciano, una asociación. Ahora bien, para ninguno de los grupos esta forma jurídica es del todo significativa, sino una forma legal de representación. Por ejemplo, en el caso navarro se hace hincapié en el estilo organizativo menos burocráticoprofesionalizado y más humano en base al organigrama que denominan como "mandala trapero". Una peculiaridad en las formas jurídicas de ambos grupos es la no existencia de empresas de inserción. En el caso navarro no existe tal posibilidad jurídica, por lo que la actividad productiva que genera empleos se articula directamente desde la propia fundación y no desde una empresa participada por ella. Por su parte, en Murcia, sí existe tal posibilidad y el grupo ha intentado crear una empresa de inserción, pero el gobierno murciano en la práctica no ha abierto tal registro de empresas de inserción, por lo que el grupo de Emaús no puede reconocerse como tal tipo de empresa ante el ministerio del gobierno central.

Respecto al liderazgo y la participación, existe un delicado equilibrio entre un liderazgo histórico carismático (sobre la figura en cada caso de la única persona restante y permanente desde el inicio del proceso), y unas formas más colectivas y colegiadas dentro de un grupo de dirección de unas cuatro o cinco personas. Al tratarse de grupos relativamente grandes y áreas de acción diferenciadas, se hace hincapié en el estilo humano y de compromiso de cada persona, por lo que no integrarían a cualquier tipo de trabajador/a externo sin ese estilo que propicie una cierta orientación de la participación.

En cuanto al crecimiento de estos grupos, puede decirse que hay una lógica combinada. Por un lado hay ciertos límites en cuanto al crecimiento de la comunidad, de ahí su carácter orgánico, mientras que en la parte laboral hay una lógica más estratégica, por ejemplo respecto a la reciente ampliación de las naves de trabajo navarras, debido a que las condiciones de mercado les obligan a evolucionar de meros recogedores a manipuladores y reutilizadores de los materiales y bienes recogidos. Cabe decir que estos grupos subrayan que 
no pretenden separar la lógica ideológica de la laboral, pero sin entrar en contradicción, sí parece que hay diferencia en la lógica de crecimiento al menos entre el ámbito comunitario y el empresarial.

En cuanto a la orientación de la acción, cabe también decir que es más plural o mixta si cabe que en los otros modelos. En el caso navarro podría decirse que coexisten las lógicas utópica y mercantil, cada una ligada a diferentes ámbitos de acción. Mientras que en el caso murciano, también ha habido ciertos rasgos asistenciales.

\subsection{Tensiones de identidad}

En el análisis de la evolución de los diferentes modelos organizativos se constató la aparición de una serie de aspectos referidos a la identidad y la legitimidad organizativa de estos modelos, y que han sido organizados en torno a varios debates comunes a todos los grupos y que son presentados en este epígrafe.

Desde el punto de vista teórico, la argumentación de este apartado se sostiene en la amplia tradición de los estudios sobre legitimidad e identidad en el contexto de las organizaciones, que ha tenido, a su vez, un desarrollo teórico específico para el caso de las empresas sociales. Con respecto a la legitimidad, partimos de la definición clásica de Schumman que la entiende como "el supuesto o la percepción generalizada de que, dado un sistema de normas, valores, creencias y definiciones socialmente construido, las acciones de una entidad son socialmente deseables, adecuadas o apropiadas" (Schumman, 1995: 574, traducción propia). Identifica además cómo las organizaciones ponen en práctica estrategias para tratar de mantener y reestablecer tres tipos de legitimidad: pragmática, moral y cognitiva. La idea subyacente es que las organizaciones necesitan ganar y mantener su legitimidad ante diferentes grupos de interés (externos e internos) para acceder a los recursos que le permitan sostenerse como organización.

Sin embargo, y según se argumenta desde la teoría institucional, la búsqueda de legitimidad realimenta el proceso denominado "isomorfismo institucional” (Di Maggio y Powell, 1983; Scott, 1992). Este concepto describe cómo las organizaciones de un determinado ámbito tienden a converger en prioridades, patrones de conducta, prácticas y estructura organizativa, hacia un modelo que se institucionaliza como "el adecuado" para ese ámbito de actividad. Siguiendo esta lógica, Dart (2004) argumenta que, en el caso de las

REVESCO $\mathrm{N}^{\circ} 113$ - MONOGRÁFICO: Crisis económica actual y sus posibles repercusiones en la economía social - ISSN: 1885-8031 - www.ucm.es/info/revesco 
Empresas Sociales, la búsqueda y mantenimiento de legitimad (particularmente legitimidad moral) en el actual contexto de predominio de valores políticos e ideológicos neoliberales, pro-mercado y pro-business, lleva a la generalización de un modelo de empresa social muy centrado en los aspectos más mercantiles, y en la adopción de formas de conducta propias de los negocios (se entiende que en contraposición a "lo social" que también forma parte de su propia definición) $)^{11}$.

Con respecto a la identidad organizativa, Albert y Whetten (1985) la definen como aquel grupo de características que los miembros de una organización consideran como “centrales, distintivas y duraderas". Gioia et al (2000) la entienden como la parte central de la cultura organizativa que permite dotar de significado a las actividades, experiencias e historias compartidas por sus miembros. En cualquier caso, existen sobre la identidad organizativa diferentes enfoques que van desde los más funcionales hasta los más críticos. Esto últimos argumentan que el proceso de construcción no es libre, sino que está condicionado por relaciones de poder y discursos dominantes que reprimen ciertas manifestaciones identitaria e imponen otras. Se cuestiona además, que el resultado de la construcción identitaria se tenga siempre efectos positivos para las personas y para la organización (Alvesson et al 2008).

En el caso de los Traperos de Emaús, el primero de los ámbitos en el que se manifiestan los debates sobre identidad y legitimidad se resumen en el que denominamos genéricamente como el debate sobre "ser una entidad social versus ser una empresa". Y es que en prácticamente todos los grupos de traperos se constata una notable resistencia a autoidentificarse como una empresa, por considerar que este es un término con una carga ideológica capitalista. Aun reconociendo explícitamente el carácter de proyecto económico de la experiencia, se prefiere construir su identidad en torno a los términos entidad social o proyecto colectivo ya que reflejan un cultura de proceso con carácter transformador.

En este sentido y particularmente en las entidades del modelo mixto, se indica cómo el separarse del término empresa ayuda a identificar el tipo de prácticas de gestión propias de las empresas de capital (y el lenguaje que las legitima) y que no debería entrar a formar parte de la cultura organizativa de los traperos de Emaús. A modo de ejemplo se mencionan cómo el

\footnotetext{
${ }^{11}$ Aun así, Nicholls y Cho (2006) plantean la necesidad de mayor investigación sobre este asunto ya que, al contrario de lo expuesto por Dart, observan cómo mediante la búsqueda de legitimidad las Empresas Sociales pueden resistir las presiones isomórficas y mantener su capacidad de generar mayor impacto social.
}

REVESCO No 113 - MONOGRÁFICO: Crisis económica actual y sus posibles repercusiones en la economía social - ISSN: 1885-8031 - www.ucm.es/info/revesco 
uso de los términos eficiencia y eficacia empresarial sirven para legitimar el prescindir de las personas en momentos de crisis, para separar vida laboral y vida personal a la hora de tomar decisiones organizativas, o para denostar el uso de mecanismos de defensa colectiva como el reparto del empleo o la redistribución salarial.

La construcción identitaria desde esta perspectiva obliga a reformular el significado de términos como eficiencia y eficacia en el contexto de organizaciones que han tomado la opción política de trabajar específicamente con personas en situaciones de pobreza y exclusión social. Todo esto obliga a un trabajo continuado de comunicación, revisión y reflexión sobre prioridades organizativas y formas de actuar, que son potenciales fuentes de conflicto con personas que entienden su actividad en Emaús como un simple empleo en el sector de recogida y reciclaje.

Sin embargo, este debate tiene a su vez una derivada que cabe analizarse desde el punto de vista de la generación de legitimidad para la organización, lo que permite obtener y mantener el acceso a recursos estratégicos para su supervivencia. Siguiendo el trabajo de Nicholls y Cho (2006), puede argumentarse que la apuesta por identificar a las organizaciones de Traperos de Emaús con la Economía Solidaria es un intento de construir y mantener legitimidad moral que les permita resistirse al isomorfismo de mercado que acabaría desequilibrando la balanza hacia el lado de los negocios en detrimento de lo social. La pertenencia a REAS ofrece una narrativa de proyecto económico alternativo, con perspectiva de transformación social, del que los grupos de traperos pueden derivar legitimidad moral frente a los grupos de interés clave.

La segunda cuestión que introduce elementos de identidad y legitimidad se refiere a lo que denominamos el debate "sobre las empresas de inserción (EI)". En este sentido, y aunque la narrativa sobre las EI ofrece una identidad común sometida a regulación jurídica y organizativa, los colectivos Emaús utilizan esta figura a la vez que expresan serias dudas sobre la lógica de la reinserción laboral. De esta forma, aunque esta lógica determina de una forma clave su estructura y devenir organizativo, se es consciente de que en las actuales circunstancias económicas la reinserción de muchas de las personas que trabajan en Emaús en el mercado laboral ordinario es poco menos que una quimera.

Aun así existen algunas diferencias entre grupos con respecto a cómo gestionar esta contradicción. Mientras que algunos colectivos como los de Bilbao y Vitoria consideran que REVESCO No 113 - MONOGRÁFICO: Crisis económica actual y sus posibles repercusiones en la economía social - ISSN: 1885-8031 - www.ucm.es/info/revesco 
el formato de empresa de inserción permite margen de maniobra para establecer una lógica de funcionamiento organizativo que consolide a la entidad y que contribuya a que al menos ciertas personas mejoren su empleabilidad, en otros colectivos como el Navarro y Murciano, la cuestión es puramente estratégica y se utiliza la legitimidad que ofrece esta figura con una elemento instrumental para la estrategia de consecución de los objetivos de acogida y transformación social que pretenden sus organizaciones.

El tercero de los aspectos que hace referencia al tema de la profesionalización, y a que la creciente necesidad de recursos financieros amenace la independencia de los grupos de traperos, se ilustra en los debates que hemos denominado como "vivir de subvenciones".

Existe una manifiesta preocupación en los colectivos de Emaús por el hecho de que la necesidad de racionalizar y profesionalizar su actividad para acceder a más recursos y multiplicar su impacto pueda acabar convirtiendo a las organizaciones en meros instrumentos de la administración y sus políticas de inserción profesional. Incidir en la separación entre subvenciones y pago por servicios prestados a administraciones públicas es una reivindicación habitual de los representantes de las organizaciones para poder así visualizar y comunicar su independencia.

La pregunta aquí tiene que ver con si es posible cambiar la orientación de las políticas de integración mediante la experiencia de la Economía Solidaria, huyendo del modelo de Empresas de Inserción puramente funcionales, sin perder la agenda trasformadora que pretenden los colectivos de Emaús. En este sentido, y frente a los debates anteriores, la cuestión reside en si el nivel de legitimidad pragmática derivada de la conformidad con estándares y resultados requeridos para justificar las políticas públicas, no se consigue a costa del peligro de ceder ante las presiones isomórficas derivadas del estado. Se manifiestan así controversias con respecto a los perfiles profesionales que la administración exige en ciertos puestos de trabajo de las empresas de inserción (independientemente de su idoneidad para la organización), la dificultad para contratar para el proyecto a la personas que la organización elija (frente a las que son derivadas de los servicios sociales de las administraciones públicas), o las dinámicas de crecimiento organizativo inducido por proyectos puntuales que pueden acabar dando al traste con las políticas de consolidación de la identidad colectiva, o las estrategias disponibles para momentos de crisis.

REVESCO No 113 - MONOGRÁFICO: Crisis económica actual y sus posibles repercusiones en la economía social - ISSN: 1885-8031 - www.ucm.es/info/revesco 


\section{RESPUESTAS A LA CRISIS}

Son varios los autores y estudios que sostienen que los actores de la economía social y solidaria reaccionan de manera diferente frente a la crisis económica en comparación con el conjunto de la economía privada. Por ejemplo, Birchall y Hammond (2009) mantienen que durante las crisis las funciones sociales de las organizaciones de la economía social se hacen más latentes, y Zevi et al. (2011) concluyen, para casos de diferentes países, que estas organizaciones evitan mejor los efectos negativos de la crisis, sorteando las diferentes complicaciones a corto plazo y consiguiendo así mantener el empleo con una visión de generación de riqueza para el largo plazo.

Por su parte, Chaves y Monzón (2012) explican que, por un lado, la economía social en el corto plazo amortigua los efectos de la crisis, y por otro lado, constituye una parte fundamental de la salida estructural a la misma. Argumentan que esa capacidad de amortiguación se deriva de algunas características propias de las organizaciones de la economía social, tales como: normas particulares (motivación sin ánimo de lucro, reglas de utilización de beneficios, doble naturaleza de sus miembros, no deslocalización de sus actividades, etc.), o compromisos sociales (modo de gobernanza y tendencia a la democracia interna, así como búsqueda de consensos que facilitarían respuestas flexibles en aspectos tales como horarios de trabajo y salarios).

En el mismo sentido, los estudios de Calderón y Calderón (2012a, 2012b) demuestran cómo los efectos negativos de la crisis sobre el empleo son menores en las organizaciones de economía social, debido a una mayor corresponsabilidad de los trabajadores en la toma de decisiones sobre la flexibilidad interna y la calidad del empleo. Estas autoras analizan cómo en los períodos de recesión la destrucción de empleo en las cooperativas es menor que en las empresas convencionales, en gran medida gracias a la adaptación de los salarios y las horas de trabajo, y también estudian cómo actúan de refugio para parte del empleo que se destruye en el resto de empresas. Pero además, las cooperativas son capaces de actuar de refugio manteniendo mejores estándares de calidad en el empleo que en la empresa privada en cuestiones como igualdad de género, seguridad, inclusión, diversidad, no discriminación, y flexibilidad.

La mejor capacidad de adaptación a las crisis, debido a la mayor flexibilidad, también es subrayada por Clemente et al. (2012). Cuestión que para el caso de las cooperativas, haría REVESCO No 113 - MONOGRÁFICO: Crisis económica actual y sus posibles repercusiones en la economía social - ISSN: 1885-8031 - www.ucm.es/info/revesco 
que la variación en el empleo resulte ser menos sensible al ciclo económico (aunque a la larga del mismo signo que el propio ciclo), actuando en cierta manera de estabilizador automático sobre el empleo (Díaz y Marcuelo, 2010). Por lo que en definitiva, las empresas de la economía social serían una buena opción estratégica para el desarrollo local y la inserción sociolaboral, la mejora en la calidad del empleo, y la cohesión social y territorial en tiempos de crisis (Melián y Campos, 2010).

Las respuestas de los grupos de Emaús a la crisis se pueden ubicar sin duda en este marco alternativo de la economía social y solidaria. Ahora bien, dentro de este amplio espectro existen diferentes situaciones y respuestas concretas, de hecho, a continuación mostramos cómo, si bien la tendencia general es la teóricamente descrita, incluso dentro de la pequeña muestra que suponen los grupos de Emaús en el Estado existen algunas diferencias.

Primero queremos aclarar que parece haber dos periodos diferenciados durante la crisis en lo que a la evolución de los grupos de Emaús se refiere, uno desde el 2008 hasta el 2011, y otro que se abre en el 2012. De modo que durante el primer periodo, a pesar de la crisis económica, existen en Emaús situaciones expansivas y de crecimiento, mientras que en el segundo periodo se hacen más necesarios otro tipo de ajustes frente a la crisis. Así que durante ese primer periodo sí puede decirse que Emaús cumple esa función de "refugio de empleo" típica de las organizaciones de la economía social, tal y como muestra el Cuadro 3 a continuación (aun siguiendo lógicas diferentes según el grupo).

Cuadro 3. Datos sobre empleo e ingresos, 2008-2011.

\begin{tabular}{|c|c|c|c|c|c|c|c|c|}
\hline & \multicolumn{4}{|l|}{2011} & \multicolumn{4}{|l|}{$2008^{(1)}$} \\
\hline & Empleo & Ingresos & $\begin{array}{l}\text { Facturación } \\
\text { Mercado\% }\end{array}$ & $\begin{array}{l}\text { Subvención } \\
\%\end{array}$ & Empleo & Ingresos & $\begin{array}{l}\text { Facturación } \\
\text { Mercado\% }\end{array}$ & $\begin{array}{l}\text { Subvención } \\
\%\end{array}$ \\
\hline Bizkaia & 95 & 5.361 .383 & 74 & 21 & 55 & 1.786 .939 & 66 & 32 \\
\hline Gipuzkoa & 115 & 5.304 .886 & 22 & 73 & 70 & 4.031 .000 & 18 & 77 \\
\hline Navarra & 206 & 4.387 .000 & 90 & 10 & 182 & 3.723 .827 & 88 & 10 \\
\hline Araba & 27 & 732.593 & 46 & 54 & 8 & 121.964 & 40 & 60 \\
\hline Sabadell & 13 & 250.00 & 100 & 0 & 11 & 180.000 & 100 & 0 \\
\hline
\end{tabular}

Fuente: Elaboración propia a través del Sistema de Auditoría Social de REAS para Euskadi y Navarra; y de cuestionario para Sabadell y Murcia.

(1) Los datos para Araba son del 2009.

REVESCO No 113 - MONOGRÁFICO: Crisis económica actual y sus posibles repercusiones en la economía social - ISSN: 1885-8031 - www.ucm.es/info/revesco 
Como podemos observar, los tres grupos correspondientes a los modelos fundación y modelo empresa social crecen espectacularmente en empleo e ingresos, Bizkaia $(72 \%, 300 \%)$ Gipuzkoa (64\%, 31\%); Araba (337\%, 600\%). Mientras que Navarra (modelo mixto), experimenta también un crecimiento, pero mucho más moderado $(10 \%, 17 \%)$; y similar, pero menor al de Sabadell (18\%, 38\%), correspondiente al modelo comunitario. Esta diferenciación, puede deberse principalmente al papel de las políticas sociales en la Comunidad Autónoma del País Vasco, y a la menor y más tardía incidencia de la crisis económica en dicha comunidad. Es importante subrayar también que todos los grupos siguen la estrategia de aumentar la facturación de mercado frente a la mera obtención de subvenciones, aunque la estructura de cada uno difiere, siendo Gipuzkoa el caso más claro en el que la gran mayoría de ingresos proviene de subvenciones, y el de Sabadell el caso extremo contrario, en el que la totalidad de ingresos en esos años concretos ha provenido de facturación de mercado.

Esta tendencia de crecimiento cambia progresivamente a partir de 2012, y en general comienzan o se agudizan algunos de los principales efectos de la crisis, y las estrategias de respuesta a estos, que presentamos a continuación, aunque las fechas exactas y la magnitud de las consecuencias varían según región.

Debido a la crisis se han reducido los ingresos, especialmente a partir de mediados del 2012, y además, la totalidad de los grupos han experimentado reducciones en los ingresos provenientes de las diversas administraciones públicas, ya sea por reducción de subvenciones, contratos, o eliminación de planes concertados.

Esta reducción de ingresos genera tensión sobre algunos proyectos propios y sobre el mantenimiento del empleo, cuestión sobre la que se observan importantes diferencias entre modelos. En primer lugar, en los grupos de Bizkaia y Araba (modelo empresa social), "sí ha habido que reducir algunos puestos de inserción, pero no puestos de la gente que lleva tiempo asociada en las cooperativas o que cubre puestos de estructura", ya que estos pueden mantenerse debido a la flexibilidad en las funciones y la bajada de salarios. En segundo lugar, en Gipuzkoa (modelo fundación), ha habido experiencias precisamente en el sentido contrario: despidos de personas antiguas y de estructura al mismo tiempo que se aceptaban nuevos puestos de inserción con el objetivo de conseguir nuevas subvenciones. Este hecho ha sido fuente de fuertes controversias tanto dentro del grupo de Gipuzkoa (siendo uno de los

REVESCO No 113 - MONOGRÁFICO: Crisis económica actual y sus posibles repercusiones en la economía social - ISSN: 1885-8031 - www.ucm.es/info/revesco 
motivos que ha llevado a su división), como entre los grupos del Estado, ya que ha perjudicado la imagen de Emaús. En tercer lugar, en Sabadell (modelo comunidad), no hay variaciones sustanciales, ya que "el tema laboral siempre se ha gestionado de forma interna y los fondos se han gestionado en comunidad", aunque sí reconocen que ahora deben cuidar más el equilibrio entre las personas cotizantes y aquellas cobrando subsidio de desempleo o sin ingresos. Por último, entre Murcia y Navarra (modelo mixto) hay ciertas diferencias, y variaciones pero no muy significativas, aunque sí hay que subrayar la apuesta por una más grande nave para el trabajo propio en Navarra que explicamos más adelante.

Frente a la reducción de ingresos, y con el objetivo general de mantener el empleo, una estrategia común a todos los grupos es la adecuada combinación de bajada de sueldos y/o la reducción de horas de trabajo, así como la flexibilidad en funciones. De todas formas, hay que subrayar que las diferencias salariales en los diferentes grupos ya eran muy bajas de antemano, quizá con la salvedad del caso de Gipuzkoa.

Otra estrategia para hacer frente a la crisis es la búsqueda de nuevas fuentes de ingresos. Por ejemplo en Bizkaia han intentado entrar en nuevos sectores de actividad (movilidad sostenible) o mejorar la calidad del servicio en tienda. En Sabadell, han procurado diversificar actividades, aunque la estrategia más clara ha consistido en abrir otra tienda para aumentar la cercanía a la clientela y el volumen de ventas. En Navarra, la estrategia es quizá más arriesgada, puesto que han realizado una gran inversión para poder abrir una nueva planta procesadora. La necesidad de esta nueva planta radica en el hecho de que debido a la crisis, se recogen materiales de menor calidad, lo que les obliga a cambiar su modelo de negocio de meros recogedores y vendedores, a manipuladores de los materiales para poder venderlos, y reutilizadores, siendo que estas nuevas secciones en la cadena de su actividad requieren de más espacio del que disponían en la planta antigua.

Por otro lado, hay dos estrategias que ya se pusieron en marcha antes de la crisis, pero que todos los grupos han subrayado como de vital importancia para hacer frente a misma. En primer lugar, la búsqueda de un mayor equilibrio en la sostenibilidad económica, a través de la reducción de la dependencia de subvenciones públicas y el aumento de facturación propia a través del mercado (con la salvedad de Gipuzkoa), que ya se ha mencionado al analizar los datos de evolución entre el 2008 y 2011. Y en segundo lugar, el establecimiento de sistemas

REVESCO No 113 - MONOGRÁFICO: Crisis económica actual y sus posibles repercusiones en la economía social - ISSN: 1885-8031 - www.ucm.es/info/revesco 
de protección colectiva para sus miembros (p.ej. de atención médica), aunque con algunas pequeñas diferencias entre modelos.

Por último, cabe reseñar que los grupos Emaús más allá de ser entidades de la economía social y solidaria consideran fundamental su labor social en muchos ámbitos no estrictamente económicos o productivos, y que en estos ámbitos también ha incidido claramente la crisis. Por ejemplo, los grupos de Navarra y Sabadell subrayan que como respuesta a las crecientes problemáticas sociales han reforzado su labor en colectivos y plataformas que luchan por la justicia social en diferentes ámbitos, y que han iniciado proyectos de atención social, montados prácticamente a coste cero con la ayuda de voluntarios.

Para acabar, respecto a los modelos organizativos, debemos señalar que en Sabadell, Murcia y Navarra parece que no se generan tensiones sobre sus organigramas y formas de funcionamiento específicamente debido a la crisis, mientras que en Bizkaia y Araba sí, ya que "todo lo laboral nos afecta directamente a lo organizativo" por tratarse de cooperativas de trabajo asociado. Por su parte, en Gipuzkoa es el propio modelo el que está en cuestión a raíz de la crisis, principalmente debido a su dependencia de financiación respecto a unas administraciones con presupuestos decrecientes y su orientación funcional a las políticas de asistencia social de las mismas.

En conclusión, no hay una tipología nítida de respuestas a la crisis que se corresponda estrictamente con los modelos organizativos, aunque la crisis sí sigue marcando las diferencias entre estos modelos, que se mantienen, tal vez con la salvedad de la quiebra del modelo en Gipuzkoa (que sin embargo no se debe solo a la crisis sino que también a otros factores de orden interno). La diferencia más clara en correspondencia con cada uno de los modelos tal vez sea la referida al impacto sobre el mantenimiento del empleo.

Así que, respecto a las variables que determinan cada modelo y su relevancia respecto a los impactos y respuestas a la crisis, podemos decir que: (i) La forma jurídica no parece una variable determinante; tal vez con la salvedad del modelo empresa social en el que la forma cooperativa resalta el impacto de lo laboral sobre lo organizativo. (ii) La existencia o no de comunidad, y las formas de liderazgo y participación, no parecen variables explicativas significativas respecto a las respuestas a la crisis, aunque haya cierta correlación con comportamientos diferenciados; tal vez con la salvedad del modelo comunitario en el que la 
total puesta en común de los recursos condiciona también las estrategias laborales (iii) el modelo de crecimiento seguido y la orientación de la acción, parecen las variables que más han marcado los impactos de la crisis sobre los grupos y sus respuestas; aunque a su vez, hay que subrayar la incidencia sobre estas variables del factor externo correspondiente a la diversidad de financiación de políticas sociales existente entre las comunidades del País Vasco y Navarra, y las de Murcia o Cataluña.

\section{CONCLUSIONES}

La primera conclusión y constatación importante es que, a pesar del nombre y filosofía originaria común, conviven en el Estado grupos de Traperos de Emaús con características múltiples muy dispares, pudiéndose identificar cuatro modelos organizativos: (i) modelo comunitario; (ii) modelo fundación; (iii) modelo empresa social; (iv) modelo mixto. Las diferencias entre modelos se pueden observar en torno a las variables de: (i) la forma jurídica de cada grupo; (ii) la existencia de vida en comunidad; (iii) las formas de liderazgo y participación, y el modelo de gobernanza; (iv) las razones para el crecimiento de la organización y la multiplicación de su impacto; (v) el carácter global de la orientación de su acción.

El segundo ámbito de conclusiones se refiere al hecho de que todos los grupos viven tensiones de identidad y legitimidad sobre sus modelos organizativos. Un primer aspecto común de estas tensiones tiene que ver con la auto-identificación como entidad social o como empresa. La mayoría de grupos son reacios a calificarse como empresa, ya que aunque su labor productivo-económica pudiera encajar en los análisis como empresas sociales, prefieren subrayar la integralidad de su labor social. El segundo aspecto se refiere a la pertinencia y modelo sobre las empresas de inserción laboral, cuestión sobre la que existen divergencias entre grupos. El tercer aspecto detectado se refiere al equilibrio entre facturación de mercado y subvenciones para garantizar su independencia y sostenibilidad económica. En este campo, casi todos los grupos salvo el de Gipuzkoa han optado por aumentar la facturación propia, a pesar de que los porcentajes varían sustancialmente entre ellos.

Por último, respecto a las respuestas a la crisis, por un lado debemos mencionar que no hemos encontrado tipologías de respuestas que se correspondan y diferencien nítidamente respecto a cada uno de los modelos organizativos, pero que, durante la crisis, sí se mantienen las diferencias entre estos modelos. Por otro lado, parece que en parte debido a la crisis, uno REVESCO No 113 - MONOGRÁFICO: Crisis económica actual y sus posibles repercusiones en la economía social - ISSN: 1885-8031 - www.ucm.es/info/revesco 
de los modelos organizativos, el denominado como fundación y que es más dependiente de las subvenciones públicas, puede quebrarse, y por tanto, tendrá que reinventarse. Para acabar, queremos insistir en que gracias a las lógicas de solidaridad interna y externa, la mayoría de grupos de Emaús presentan rasgos de respuesta a la crisis evidentemente diferentes a las de la economía privada, con una clara apuesta por el mantenimiento del empleo utilizando para ello estrategias de flexibilidad y compromiso laboral.

\section{BIBLIOGRAFÍA}

ALBERT, S. y WHETTEN, D.A. (1985), Organizational identity. Research in organizational behaviour, $n^{o} 7$, pp.263-295.

ALVESSON, M., ASHCRAFT, K.L. y THOMAS, R. (2008), Identity Matters: Reflections on the Construction of Identity Scholarship in Organization Studies. Organization, vol.15, $\mathrm{n}^{\mathrm{o}} 1, \mathrm{p} 5-28$.

BIRCHALL, J. y HAMMOND, L. (2009), Resilience of the cooperative business model in times of crisis. ILO-Sustainable Enterprise Programme. ISBN: 978-92-2-122409-9

BISHOP, M. y GREEN, M. (2008), Philantrocapitalism: How the rich can save the World. New York: Bloomsbury Pres. ISBN: 10-1596913746.

CALDERÓN, B. y CALDERÓN, M.J. (2012), La calidad del empleo de las entidades de la Economía Social en períodos de crisis. Ekonomiaz, no 79, 1er cuatrimestre, pp.31-57.

CALDERÓN, B. y CALDERÓN, M.J. (2012), Cómo afrontan la crisis las cooperativas en España: comparativa de trayectorias laborales a partir de la Muestra Continua de Vidas Laborales. CIRIEC-España, Revista de Economía Pública, Social y Cooperativa, ${ }^{\circ} 76$, diciembre, pp.5-26.

CHAVES, R. y MONZÓN, J. L. (2012), The Social Economy in the European Union. CIRIEC. EESC European Economic and Social Committee.

ClEMENTE, J., DÍAZ-FONCEA, M., MARCUEllo, C. y SANSO-NAVARRO, M. (2012), The wage gap between cooperative and capitalist firms: evidence from Spain. Annals of Public and Cooperative Economics, vol.83, n³, septiembre 2012, pp.337-356.

CORAGGIO, J. L. (2011), Economía Social y Solidaria. El trabajo antes que el capital. Ecuador: Abya-Yala/UPS Publicaciones. ISBN: 978-9978-22-999-6.

DA ROS, G.S. (2007), Economía solidaria: aspectos teóricos y experiencias. Unircoop, vol.5, n'1, pp. 9-27.

REVESCO No 113 - MONOGRÁFICO: Crisis económica actual y sus posibles repercusiones en la economía social - ISSN: 1885-8031 - www.ucm.es/info/revesco 
DART, R. (2004), The Legitimacy of Social Enterprise. Nonprofit Management and Leadership, vol.14, $\mathrm{n}^{\circ} 2$, pp. 411-424.

DEFOURNY, J. y NYSSENS, M. (2006), Defining social enterprise. En: NYSSENS M., (eds) Social enterprise: At the crossroads of market, public policies and civil society. Abingdon: Routledge, p. 3-26. ISBN. 9780415378789.

DEFOURNY, J. y NYSSENS, M. (2010), Conceptions of Social Enterprise and Social Entrepreneurship in Europe and the United States: Convergences and Differences. Journal of Social Entrepreneurship, vol. 1, n¹, pp. 32-53.

DEY, P. y STEYAERT, C. (2010), The politics of narrating social entrepreneurship. Journal of Enterprising Communities: People and Places in the Global Economy, vol. 4, $\mathrm{n}^{\circ} 1$, pp. 85-108.

DÍAZ, M. y MARCUELLO, C. (2010), Impacto económico de las cooperativas. La generación de empleo en las sociedades cooperativas y su relación con el PIB. CIRIECEspaña, Revista de Economía Pública, Social y Cooperativa, nº 67, abril 2010, pp.23-44.

DIMAGGIO, P. y POWELL, W. (1983), The Iron Cage Revisited: Institutional Isomorphism and Collective Rationality in Organizational Fields. American Sociological Review, $\mathrm{n}^{\mathbf{0}} 48$, pp. 147-160.

ENJOLRAS, B. (2010), The Public Policy Paradox. Normative Foundations of Social Economy and Public Policies Which Consequences for Evaluation Strategies?. En: Bouchard M.J. (eds). The Worth of the Social Economy. An International Perspective. Bruxelles: Peter Lang. pp.43-60. ISBN: 978-90-5201-580-4.

GIOIA, D.A., MAJKEN, S. y CORLEY K.G. (2000), Organizational identity, image, and adaptive instability. The Academy of Management Review, vol. 25, nº1, pp. 63-81.

GURIDI, L., PEREZ DE MENDIGUREN, J. C., IAMETTI, A., DEUX, M.V., VAZQUEZ, G. y URIBE, A. (2011), Experiencias de Economía Social y Solidaria: compartiendo estrategias y aprendizajes. Papeles de Economía Solidaria (en línea), nº 2, pp.1-77. Disponible en Internet: http://www.economiasolidaria.org/papeles_ES_2.

KERLIN, J. A. (2006), Social Enterprise in the United States and Europe: Understanding and Learning from the Differences. Voluntas, vol 17, n 3, pp. 246-262.

KERLIN, J. A. (2010), A Comparative Analysis of the Global Emergence of Social Enterprise. Voluntas, vol. 21, nº2, pp. 162-179.

LAVILLE, J. L. (2004), El marco conceptual de la Economía Social y Solidaria. En: Laville J.L. (eds) Economía social y solidaria. Una visión europea. Buenos Aires: Fundación REVESCO No 113 - MONOGRÁFICO: Crisis económica actual y sus posibles repercusiones en la economía social - ISSN: 1885-8031 - www.ucm.es/info/revesco 
OSDE - Universidad Nacional de General Sarmiento - Editorial Altamira. pp. 1-25. ISBN: 9879423348.

MARTINEZ, A. y ALVAREZ, S. (2008), La economía crítica y solidaria: perspectivas teóricas y experiencias para la construcción de una economía alternativa. In: Worldwatch Institute (eds). La situación del mundo 2008. Innovaciones para una economía sostenible. Informe anual del Worldwatch Institute sobre el Progreso hace una Sociedad Sostenible. Barcelona: CIP-Ecosocial e Icaria, pp. 371-486. ISBN: 84-7426-971-0.

MELIÁN, A. y CAMPOS, V. (2010), Emprendedurismo y economía social como mecanismos de inserción sociolaboral en tiempos de crisis. REVESCO, Revista de Estudios Cooperativos, $\mathrm{n}^{\circ} 100$, Extraordinario, pp.43-67.

NICHOLLS, A. (2010), The Legitimacy of Social Entrepreneurship: Reflexive Isomorphism in a Pre-Paradigmatic Field. Entrepreneurship Theory and Practice, n⿳30, abril 2010, pp. 611-633.

NICHOLLS, A. y CHO, A. (2006), Social entrepreneurship: The structuration of a field. En: Nicholls A., (eds) Social entrepreneurship: New models of sustainable social change. New York: Oxford University Press, pp. 99-118. ISBN: 10-0199283885.

PEATTIE, K. y MORLEY, A. (2008), Eight paradoxes of the social enterprise research agenda. Social Enterprise Journal, no 4, febrero 2008, pp. 91-107.

PEREZ DE MENDIGUREN, J. C., ETXEZARRETA, E. y GURIDI, L. (2009), Economía Social, Empresa Social y Economía Solidaria: diferentes conceptos para un mismo debate. Papeles de Economía Solidaria (en línea), $\mathrm{n}^{\circ} 1$, pp. 1-41. Disponible en internet: http://www.economiasolidaria.org/papeles_ES_1.

PORTER, M. y KRAMER, M. (2011), Creating Shared Value. How to reinvent capitalism and unleash a wave of innovation and growth. Harvard Business Review, enero-febrero 2011, pp. 1-17.

RIDLEY-DUFF, R. y BULL, M. (2011), Understanding Social Enterprise: Theory and Practice. London: Sage Publications. ISBN: 10- 1848601999.

SCOTT, W. R. (2002), Organization: Rational, Natural, and Open Systems. Englewood Cliffs, New Jersey: Prentice Hall Inc. ISBN: 10-013016559X

SPEAR, R. (2006), Social entrepreneurship: a different model? International Journal of Social Economics, vol. 33, nº 5/6, pp. $399-410$.

SUCHMAN, M. (1995), Managing legitimacy: strategic and institutional approaches. Academy of Management Review, vol. 20, n³, pp. 571-610.

REVESCO No 113 - MONOGRÁFICO: Crisis económica actual y sus posibles repercusiones en la economía social - ISSN: 1885-8031 - www.ucm.es/info/revesco 
TEASDALE, S. (2012), What's in a name? Making sense of social enterprise discourses. Public Policy and Administration, vol.27, n², pp. 99-119.

ZEVI, A., ZANOTTI, A., SOUlAGE, F. y ZELAIA, A. (2011), Más allá de la crisis: Cooperativas, Trabajo, Finanzas. Generación de riqueza para el largo plazo. CECOP. 